\title{
Review of: "Mortality and microbial diversity after allogeneic hematopoietic stem cell transplantation: secondary analysis of a randomized nutritional intervention trial"
}

\section{Erden Atilla}

Potential competing interests: The author(s) declared that no potential competing interests exist.

Patients undergoing allogeneic hematopoietic stem cell transplantation demonstrate gut mucosal barrier changes which is associated with complications and poor clinical outcomes. Interplay between microbiota and aGVHD as well as survival in allogeneic stem cell transplantation is a hot topic in recent years (Nguyen, Current Opinion in Genetics and Development, 2021). This study entitled 'Mortality and microbial diversity after allogeneic hematopoietic stem cell transplantation:secondary analysis of a randomised nutritional intervention trial' is a secondary analysis of a randomised cohort that previously published. The authors stated solid aim of this study is to show the impact of the nutritional intervention on microbiota, fecal SCFAs and biomarkers of gut barrier function as well as the impact of microbiota, SCFAs and biomarkers of gut barrier functions on survival and aGVHD. The results comprise well designed- detailed analysis of gut microbiota, short-chain fatty acids and markers of gut barrier functions of 47 patients (intervention group $n=23$; control group $n=24$ with similar characteristics) at baseline and and 3 weeks postHSCT. The authors concluded that the nutritional intervention given had no effect on the microbiota, SCFAs and markers of gut barrier functions. There are some limitations listed in the article which are reasonable: 1 . The cohort is too limited 2 . The study did not compare the route of nutritional support 3. Oral intake can be different from control and intervention groups. There are some other points to consider about the article:

1. Energy and protein intakes were reported in this current study. As described SCFAs such as butyrate, propionate and acetate are produces as a result of the fermentation of dietary fiber by the intestinal microbioata. The study design may be re-planned accordingly in future trials since all patients received fibre-free formula.

2. The study had two time points in the study (baseline or 3 weeks post transplant). The authors found that low bacterial diversity at 3 weeks and relative loss of diversity until 3 weeks as well as low abundance of Blautia, independent of pre-HSCT levels, was associated with death within the first year, but with no impact of aGVHD. Recently when the follow-up is longer Ingham et al demonstrated a clear pattern of depletion of fecal Blautia spp. immediately post HSCT, as well as their recovery from month +3 post HSCT onwards Blautia spp. abundances as an indicator of favorable clinical outcomes. Longer follow-up 
time in future trials might indicate prominent results.

3. Although this is a nutritional intervention study, it will be important to gain a more mechanistic understanding of the possible effects of antimicrobial treatment. Each antibiotic effects microbiota on different ways. Here, patients received ceftazidime or pipereacillin-tazobactam and meropenem in combination with anti fungal treatment with similar median days of administration in intervention and control group. However, the antibiotic schedule administered to each patient is not the same and so that it is hard to reach a definite conclusion about the effect of antibiotics on microbial diversity, SCFAs and markers of gut barrier functions.

4. The role of the intestinal microbiota in allo-HCT outcome have utilised 16SrRNA gene amplicon sequencing. The disadvantage of this technique is the lack of taxonomic resolution at species and strain-specific levels (Janda, J Clin Microbiol 2007). To overcome this challenge, whole-genome metagenomic sequencing can be performed for high-resolution profiles of the intestinal bacterial compositions (Fang, Front Microbiol 2018; Vatanen, Nature, 2018). 